\title{
A phase II study of neoadjuvant chemotherapy with docetaxel, cisplatin, and S-1, followed by gastrectomy with D2 lymph node dissection for high-risk advanced gastric cancer: results of the KDOG1001 trial
}

\author{
Kei Hosoda 1 - Mizutomo Azuma ${ }^{2} \cdot$ Chikatoshi Katada $^{2} \cdot$ Hiromitsu Moriya $^{1} \cdot$ Hiroaki Mieno $^{1} \cdot$ Kenji Ishido $^{2}$. \\ Akira Ema ${ }^{1} \cdot$ Hideki Ushiku ${ }^{1} \cdot$ Takuya Wada $^{2} \cdot$ Marie Washio $^{1} \cdot$ Akinori Watanabe $^{2} \cdot$ Katsuhiko Higuchi $^{2}$. \\ Satoshi Tanabe ${ }^{2}$. Wasaburo Koizumi ${ }^{2}$ Masahiko Watanabe ${ }^{1} \cdot$ Keishi Yamashita $^{1,3}$
}

Received: 10 August 2018 / Accepted: 29 September 2018 / Published online: 3 October 2018

(C) The International Gastric Cancer Association and The Japanese Gastric Cancer Association 2018

\begin{abstract}
Background The prognosis of patients with gastric cancer with bulky node metastasis, linitis plastica (type 4), or large ulceroinvasive-type tumors (type 3 ) remains poor. We conducted a phase II study to evaluate the safety and efficacy of neoadjuvant chemotherapy with docetaxel, cisplatin, and S-1 (DCS) for establishing a new treatment modality that improves prognosis. Methods Patients received up to four 28-day cycles of DCS therapy (docetaxel at $40 \mathrm{mg} / \mathrm{m}^{2}$, cisplatin at $60 \mathrm{mg} / \mathrm{m}^{2}$ on day 1 , and S-1 at $40 \mathrm{mg} / \mathrm{m}^{2}$ twice daily for 2 weeks) followed by gastrectomy with D2 nodal dissection. S-1 chemotherapy was administered for 1 year after surgical resection. The primary endpoint was the percentage of complete resections of the primary tumor with clear margins (R0 resection). The planned sample size was 40; this was calculated based on an expected R0 rate of $85 \%$ and a threshold R0 rate of $65 \%$, with a one-sided alpha of 5\% and a power of $90 \%$.

Results Between 2010 and 2017, 40 patients were enrolled. The R0 resection rate was $90 \%$. The most common grade 3 or 4 adverse events during DCS therapy were leukocytopenia (27.5\%), neutropenia (55.0\%), and hyponatremia (22.5\%). The most common grade 3 or 4 surgical morbidity was pancreatic fistula (12.5\%); mortality was $0 \%$. The pathological response rate was $57.5 \%$ with a grade 3 histological response rate of $8 \%$.

Conclusions Neoadjuvant chemotherapy with DCS was feasible and showed a sufficient R0 resection rate. A future study with a sufficient follow-up period should confirm survival outcomes.
\end{abstract}

Keywords Adjuvant chemotherapy · Gastrectomy $\cdot$ Stomach neoplasm $\cdot$ Lymphatic metastasis $\cdot$ Linitis plastica

\section{Introduction}

Kei Hosoda

k.hosoda@kitasato-u.ac.jp

1 Department of Surgery, Kitasato University School of Medicine, Kitasato 1-15-1, Minami-ku, Sagamihara 252-0374, Japan

2 Department of Gastroenterology, Kitasato University School of Medicine, Sagamihara, Japan

3 Division of Advanced Surgical Oncology, Research and Development Center for New Medical Frontiers, Kitasato University School of Medicine, Sagamihara, Japan
Gastric cancer is the fifth most common cancer and the third leading cause of cancer-related deaths worldwide [1]. Curative treatment of gastric cancer requires surgical resection. However, even when the tumor can be resected, the prognosis remains poor, particularly in those with high risk of recurrence, i.e., those with extensive lymph node metastasis (ELM) such as bulky lymph nodes along the celiac, splenic, common hepatic, or proper hepatic arteries (bulky N2 lymph nodes); linitis plastica (type 4); or large ulcero-invasive-type (type 3) [2-4].

Those with a high risk of recurrence have been the target of clinical trials. The Japan Clinical Oncology Group (JCOG) has conducted two phase II trials (JCOG0001 [2] 
and JCOG0405 [4]) to evaluate the safety and efficacy of preoperative chemotherapy followed by gastrectomy with extended node dissection for gastric cancer with ELM including bulky N2 lymph nodes. In JCOG0001, patients received irinotecan and cisplatin therapy followed by surgery. This study showed a good 3-year survival of $27.0 \%$ but was terminated because of 3 treatment-related deaths among the 55 enrolled patients. In JCOG0405, patients received two or three cycles of cisplatin and S-1 (CS) chemotherapy and then underwent surgery. The trial exhibited good feasibility, with an excellent clinical response rate (RR) of $64.7 \%$ and a 3-year survival rate of $58.8 \%$, with no treatmentrelated deaths. The JCOG also conducted a phase II trial (JCOG0210 [3]) to evaluate the safety and efficacy of preoperative CS chemotherapy followed by gastrectomy with D2 node dissection for gastric cancer with a type 4 or large type 3 tumor. The study showed good feasibility with a good 3 -year survival rate of $24.5 \%$. Based on these results, the next phase III study (JCOG0501) was conducted.

Docetaxel-containing regimens have been considered worthy of evaluating in patients with gastric cancer with ELM because the addition of docetaxel to agents containing cisplatin and 5-fluorouracil was shown to improve the survival outcomes of patients with unresectable or recurrent gastric cancer [5, 6]. However, docetaxel is not widely accepted as the standard treatment modality because of its severe toxicity. Thus, we conducted the first phase II trial (KDOG1001) of neoadjuvant chemotherapy with docetaxel, cisplatin, and S-1 (DCS) to evaluate its safety and efficacy in patients with gastric cancer with bulky N2 lymph nodes, linitis plastica (type 4) tumors, or large ulcero-invasive-type (type 3 ) tumors.

\section{Methods}

\section{Study design and population}

KDOG1001 was conducted as a prospective, single-institution, phase II trial at the Kitasato University, Tokyo, Japan. The study protocol was approved by The Kitasato University School of Medicine Research Ethics Committee and was conducted in accordance with the Declaration of Helsinki as well as the Japanese Ethical Guidelines for Clinical Studies. It was registered with the University Hospital Medical Information Network Clinical Trials Registry (http://www. umin.ac.jp/ctr/) as UMIN 000003642. All enrolled patients provided written informed consent.

The eligibility criteria were, (1) histologically proven and clinically resectable gastric adenocarcinoma (cM0); (2) bulky N2 lymph nodes (one larger than $3 \mathrm{~cm}$ or two larger than $1.5 \mathrm{~cm}$ along the celiac, splenic, common, or proper hepatic arteries), type 3 measuring $\geq 8 \mathrm{~cm}$, or type
4; (3) esophageal invasion of $3 \mathrm{~cm}$ or less; (4) an age of 20-75 years; (5) an Eastern Cooperative Oncology Group performance status $0-1$; (6) no history of prior chemotherapy, radiation therapy, or surgery for gastric cancer; (7) an adequate oral intake without any active bleeding or intestinal obstruction; (8) sufficient organ function [white blood cell (WBC) count $\geq 3000 / \mathrm{mm}^{3}$ and $\leq 12,000 / \mathrm{mm}^{3}$; hemoglobin level $\geq 8.0 \mathrm{~g} / \mathrm{dL}$; platelet count $\geq 100,000 / \mathrm{mm}^{3}$; aspartate aminotransferase (AST) and alanine aminotransferase (ALT) levels $\leq 100 \mathrm{IU} / \mathrm{L}$; total bilirubin level $\leq 1.5 \mathrm{mg} / \mathrm{dL}$; creatinine clearance $\geq 60 \mathrm{~mL} / \mathrm{min}$ in the Cockcroft-Gault equation]; and (9) written informed consent. The exclusion criteria were, (1) synchronous or metachronous (within 5 years) malignancy other than carcinoma in situ; (2) pregnant or breastfeeding women; (3) severe mental disease; (4) undergoing long-term treatment with systemic steroids; (5) undergoing treatment with flucytosine, phenytoin, or warfarin; (6) pulmonary fibrosis, interstitial pneumonitis, bowel obstruction, or ischemic heart disease requiring therapy; (7) history of severe drug allergy; (8) active infections; and (9) history of myocardial infarction within 6 months.

Initially, the absence of peritoneal dissemination and the absence of carcinoma cells on the peritoneal cytology test were confirmed via staging laparoscopy before entry into the study. However, patient accrual was so poor that we amended the study protocol at 2013 to include patients with carcinoma cells on the peritoneal cytology test (CY1). Chest radiography, contrast-enhanced thoracic/abdominal/pelvic computed tomography (CT), and upper gastrointestinal tract endoscopy were conducted as the pretreatment workup.

\section{Neoadjuvant chemotherapy}

Patients received an infusion of docetaxel $\left(40 \mathrm{mg} / \mathrm{m}^{2}\right)$ and cisplatin $\left(60 \mathrm{mg} / \mathrm{m}^{2}\right)$ on day 1 , while $\mathrm{S}-1\left(40 \mathrm{mg} / \mathrm{m}^{2}\right)$ was administered orally twice daily for 2 weeks on days $1-14$, followed by a 2 -week rest. Patients were assessed by physical examinations and laboratory tests every 2 weeks during neoadjuvant chemotherapy (NAC).

Toxicity was assessed according to the Common Terminology Criteria for Adverse Events, version 3.0. The subsequent chemotherapy cycle was delayed until patient recovery for those with severe adverse events, including the following parameters: neutrophil count $\geq 1000 / \mathrm{mm}^{3}$, hemoglobin level $\geq 7.0 \mathrm{~g} / \mathrm{dL}$, platelet count $\geq 50,000 / \mathrm{mm}^{3}$, AST and ALT levels $\leq 150 \mathrm{IU} / \mathrm{L}$, total bilirubin level $\leq 2 \mathrm{mg} / \mathrm{dL}$, and creatinine level $\leq 1.2 \mathrm{mg} / \mathrm{dL}$. To prevent life-threatening adverse events, a non-hematological adverse event of diarrhea had to be grade 0 or 1 , while the other non-hematological adverse events had to be grade 2 or lower except for nausea, vomiting, anorexia, and fatigue.

The DCS doses were reduced in the event of grade 4 leukopenia, grade 4 neutropenia, grade 4 thrombocytopenia, 
creatinine $>1.5 \mathrm{mg} / \mathrm{dL}$, or grade 3 non-hematological adverse events including febrile neutropenia.

After the first and second cycles of DCS NAC, efficacy was evaluated based on contrast-enhanced CT findings and tumor marker levels. If the tumor obviously progressed but remained resectable after the first cycle of chemotherapy or if the tumor remained stable without a marginal response after the second cycle of chemotherapy, surgical resection was performed. Otherwise, 4 cycles of the DCS therapy was given to the patients, followed by surgical resection. Surgical resection was performed between 15 and 42 days after the last administration of S-1. If curative resection was considered difficult, the protocol treatment was terminated.

\section{Surgery}

R0 resection was considered possible when, (1) resectability was assessed comprehensively with CT, an upper gastroenterological endoscopy, and a barium meal study and (2) the patient had sufficient bone marrow function (WBC $>3000$ / $\mathrm{mm}^{3}$ and a platelet count $>100,000 / \mathrm{mm}^{3}$ ). Patients who fulfilled these criteria underwent surgery between 15 and 42 days after the last administration of S-1.

After laparotomy, intraperitoneal washing cytology specimens were examined. If the cytology findings were negative, R0 resection was attempted via total or distal gastrectomy with D2 or more extended dissection, as defined by the Japanese gastric cancer treatment guidelines [7]. If $\mathrm{R} 0$ resection was considered impossible, the protocol treatment was terminated. Curative resection (R0) was defined as the removal of all macroscopic and microscopic disease. $\mathrm{R} 1$ resection was defined as the macroscopic removal of the tumor but with microscopic presence of residual tumor (positive resection margin or CY1). CY1 was defined by a positive result for carcinoma cells on the peritoneal cytology test.

\section{Postoperative chemotherapy}

S-1 therapy was started within 42 days after surgery when R0 resection was achieved pathologically. A 6-week cycle consisting of 4 weeks of oral administration of S-1 at a dosage of $40 \mathrm{mg} / \mathrm{m}^{2}$ twice daily followed by 2 weeks rest during the first year after surgery was recommended based on the third edition of the Japanese gastric cancer treatment guidelines [12]. If S-1 therapy was not started within 3 months after surgery for any reason, the protocol treatment was terminated. The protocol treatment was completed when a patient had received one or more courses of DCS NAC, had undergone R0 resection via gastrectomy with lymph node dissection, and had received postoperative chemotherapy. After completion of the protocol, no further treatment was given until tumor recurrence.

\section{Study design and statistical analysis}

This trial was designed to evaluate the safety and efficacy of DCS NAC followed by gastrectomy with D2 dissection and postoperative $\mathrm{S}-1$ therapy. The primary endpoint was the $\mathrm{R} 0$ resection rate. The secondary endpoints were the 3 -year survival rate, completion rate of the protocol treatment, pathological RR of DCS NAC, and adverse events. The pathological response was graded by the institutional pathologists according to the Japanese classification of gastric carcinoma, third English edition [8]: grade 1a, viable tumor cells occupy more than two-thirds of the tumorous area; grade $1 \mathrm{~b}$, more than one-third but less than two-thirds; grade 2, less than one-third; grade 3, no viable tumor cells. In this study, the pathological response was defined as grade $1 \mathrm{~b}$ to grade 3 responses.

Because the R0 resection rates in the JCOG0210 and the JCOG0405 were $73 \%$ and $82.4 \%$, respectively, and the efficacy of DCS therapy was expected to be superior to that of CS therapy, we hypothesized that the expected R0 resection rate was $85 \%$, which is above the $\mathrm{R} 0$ resection rate of JCOG0405 study, where the patients must be CY0. R0 resection rate of the JCOG0001 trial which evaluated the efficacy of neoadjuvant irinotecan and cisplatin chemotherapy was $65.5 \%$, where the patients must be CY0, either. Because the $\mathrm{R} 0$ resection rate after DCS NAC should be superior to that of the former JCOG trials, we set the threshold R0 resection rate to be $65 \%$. The sample size was calculated to be 40 cases with one-sided testing at the 5\% significance level with power of $90 \%$.

\section{Results}

\section{Patient characteristics}

Between May 2010 and January 2017, 40 patients were enrolled in the study. Table 1 shows the patient and tumor characteristics. The median age of the patients was 63.5 years, and the male:female ratio was $26: 14$. Seven patients had bulky N2 lymph nodes, 18 had a type 4 tumor, and 16 had a large type 3 tumor with 1 having a large type 3 tumor and bulky N2 lymph nodes. Four patients had positive cytology test at the initial staging laparoscopy.

\section{Neoadjuvant chemotherapy}

No patient had progressive disease after one cycle of DCS NAC, but one patient refused the second cycle of DCS NAC and underwent surgery because of adverse events (Fig. 1). Ten patients had stable disease after 2 cycles of DCS NAC; of these, 9 underwent surgery without additional NAC. The remaining patient received one additional cycle of NAC 
Table 1 Patient $(n=40)$ and tumor characteristics

\begin{tabular}{|c|c|}
\hline & Values \\
\hline Age $\left(\right.$ years) ${ }^{\mathrm{a}}$ & $63.5(32-75)$ \\
\hline Sex (male) & 26 \\
\hline \multicolumn{2}{|l|}{ ECOG performance status } \\
\hline 0 & 29 \\
\hline 1 & 11 \\
\hline \multicolumn{2}{|l|}{ Cancer type } \\
\hline Bulky N2 lymph nodes ${ }^{\mathrm{b}}$ & 7 \\
\hline Type 4 & 18 \\
\hline Large type $3^{b}$ & 16 \\
\hline \multicolumn{2}{|l|}{ Histological type } \\
\hline Differentiated & 7 \\
\hline Undifferentiated & 33 \\
\hline \multicolumn{2}{|l|}{ Tumor depth } \\
\hline cT3 & 7 \\
\hline cT4a & 32 \\
\hline $\mathrm{cT} 4 \mathrm{~b}$ & 1 \\
\hline \multicolumn{2}{|l|}{ Lymph node metastases } \\
\hline cNO & 18 \\
\hline $\mathrm{cN} 1$ & 11 \\
\hline $\mathrm{cN} 2$ & 10 \\
\hline $\mathrm{cN} 3$ & 1 \\
\hline \multicolumn{2}{|l|}{ cStage } \\
\hline IIA & 1 \\
\hline IIB & 20 \\
\hline IIIA & 10 \\
\hline IIIB & 8 \\
\hline IIIC & 1 \\
\hline
\end{tabular}

TNM categories are based on the third English edition of Japanese classification of gastric carcinoma

ECOG Eastern Cooperative Oncology Group

${ }^{\text {a }}$ The median is given, with the range in parentheses

${ }^{\mathrm{b}}$ Including one patient having both a large type3 tumor and a bulky N2 lymph node

and underwent surgery because of a scheduling problem. Twenty-nine patients (72.5\%) responded after 2 cycles of DCS NAC; of these, 25 received 2 additional cycles of the DCS regimen and underwent surgery. The remaining 3 and 1 patient underwent surgery without additional NAC and with an additional 1 cycle of NAC, respectively, because of adverse events.

Grade 3 or 4 toxicity during DCS NAC among the 40 treated patients included leukopenia (27.5\%), neutropenia $(55.0 \%)$, anemia (12.5\%), thrombocytopenia ( $0 \%)$, hyponatremia $(22.5 \%)$, anorexia $(20.0 \%)$, diarrhea $(10.0 \%)$, nausea (15.0\%), and febrile neutropenia (7.5\%) (Table 2). There were no chemotherapy-related deaths. The mean percentages of the actual/planned dose of docetaxel, cisplatin, and S-1 in each patient were $97.1 \%, 94.6 \%$, and $95.0 \%$, respectively.

\section{Surgical and pathological findings}

All included patients underwent surgery. R0 and R1 resection were achieved in 36 and 4 patients, respectively. The reasons for $\mathrm{R} 1$ resection were a microscopically cancerpositive distal margin in 3 patients and CY 1 in 1 patient. The R0 resection rate (primary endpoint) was $90.0 \%$ (95\% confidence interval: 76.9-96.0\%). The null hypothesis was rejected (one-sided $p<0.001$ ). The subjects included in the analysis of the primary endpoint are all registered patients. The R0 resection rates of the patients with bulky N2 lymph nodes and those with large type 3 or type 4 tumors were $100 \%$ (7/7) and $88 \%$ (30/34), respectively. The R0 resection rate in the patient population who underwent DCS for more than 2 cycles was $85.2 \%$ (23/27).

The surgical data are summarized in Table 3 . Two cases (15.2\%) were pathologically staged as pM1; one was CY1 and the other peritoneal dissemination within the bursa omentalis (Table 4). The pathological RR for primary tumors in the eligible patients was $57.5 \%$ (23/40), including 3 complete remission. A pathological response was seen in only $33.3 \%$ (4/12) of the patients receiving 2 cycles of DCS NAC; by contrast, it was seen in 69.5\% (16/23) of the patients receiving 4 cycles of DCS NAC. In patients receiving 4 cycles of DCS therapy, the pathological RR was similar for diffuse-type and intestinal-type tumor [72.2\% (13/18) and 71.4\% (5/7), respectively].

Morbidity after surgery is summarized in Table 5. Grade 3 or 4 adverse events occurred in 9 patients (22.5\%). Three repeat surgeries were performed because of smallintestinal obstruction $(n=2)$ and postoperative hemorrhage $(n=1)$. No treatment-related deaths occurred.

\section{Postoperative chemotherapy}

Of the 40 patients, 32 (80\%) subsequently began postoperative chemotherapy as a treatment protocol. Postoperative chemotherapy was not started in the remaining 8 patients due to refusal $(n=4)$, disease progression $(n=2)$, and postoperative complications $(n=2$; grade 3 pancreatic fistula and grade 2 ileus). Of the 32 patients who started postoperative chemotherapy, 25 (78.1\%) completed postoperative S-1 therapy for 1 year. Therefore, the completion rate of the protocol treatment comprising neoadjuvant DCS, surgical resection, and postoperative S1 was $62.5 \%$ (25/40). 
Fig. 1 Patient flowchart

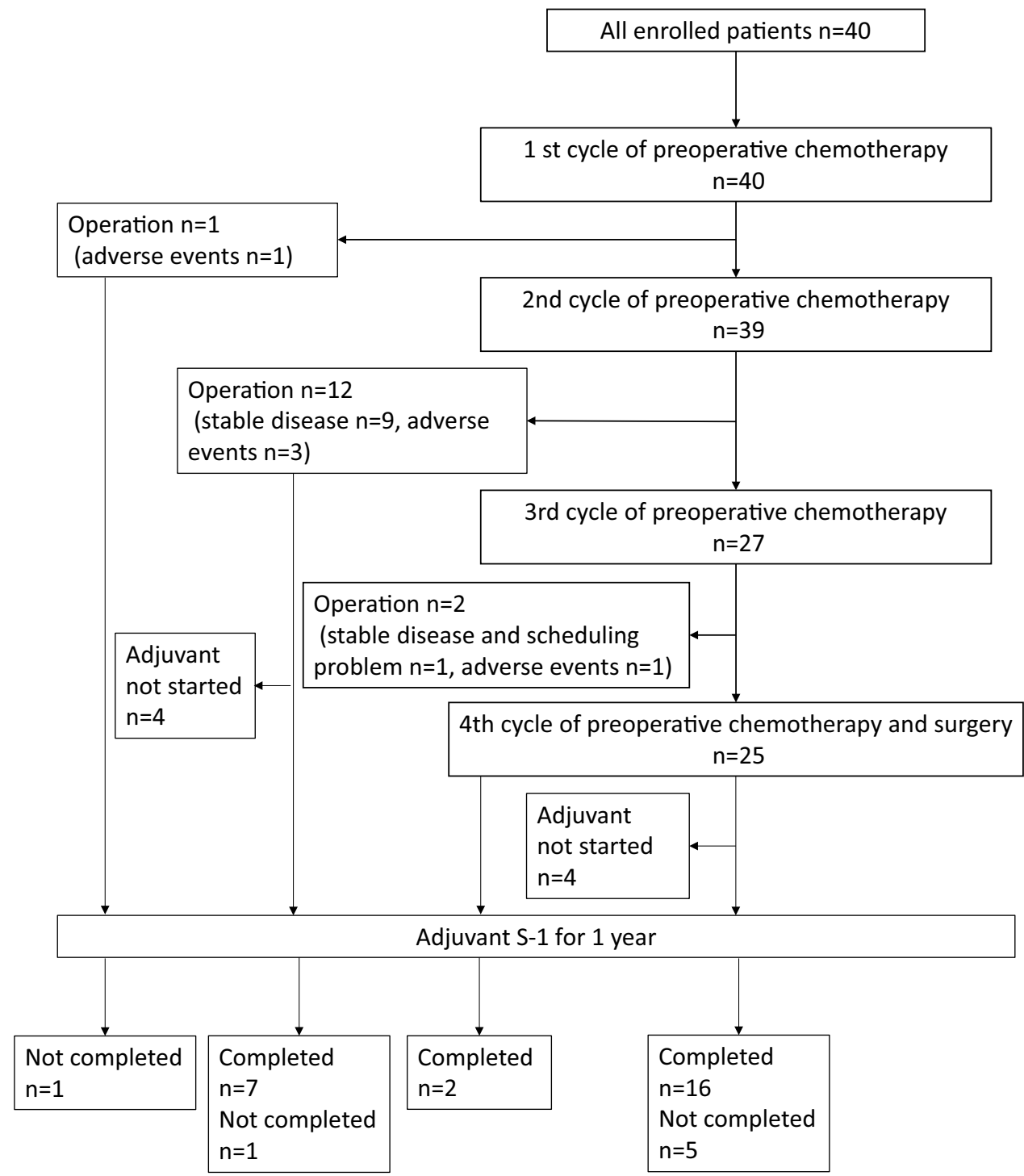

\section{Discussion}

The present trial showed an excellent $\mathrm{R} 0$ resection rate of $90 \%$, which is higher than the expected rate of $85 \%$, with acceptable adverse events. The results of the present study show that DCS NAC followed by gastrectomy is safe and feasible, warranting a subsequent phase III study.

Despite recent advances in chemotherapy, the prognosis of advanced gastric cancer remains poor. The prognosis significantly differs based on the Borrmann's macroscopic classification of gastric cancers [9-11], which includes type 1 (polypoid tumors that are sharply demarcated from the surrounding mucosa) and type 2 (ulcerated tumors with raised margins surrounded by a thickened gastric wall with clear margins) tumors. Patients with large type 3 and type 4 tumors have significantly worse prognoses than those with type 1 and 2 tumors [11]. The prognosis of patients with bulky N2 lymph nodes is also poor, and this condition is considered surgically incurable in Western countries. Large type 3 or type 4 gastric tumors or gastric cancer with bulky $\mathrm{N} 2$ lymph nodes often involve the spleen or pancreas, requiring resection of these organs. The recovery from such surgery is often prolonged, and adjuvant therapy is sometimes delayed or cancelled. Therefore, NAC is warranted in the treatment of these patients.

A randomized controlled trial showed that perioperative chemotherapy with epirubicin, cisplatin, and fluorouracil (ECF) improved overall and progression-free survival in patients with resectable gastric cancer when compared to surgery alone [12]. The $\mathrm{R} 0$ resection rate in their study was $69.3 \%$, which is much lower than our excellent outcome (R0 of $90 \%$ ). Epirubicin is not available for gastric cancer treatment in Japan. However, a triplet regimen including cisplatin and 5-FU seemed promising for neoadjuvant chemotherapy in high-risk gastric cancer. We previously conducted a phase II trial to evaluate the safety and efficacy of DCS therapy 
Table 2 Adverse events during the chemotherapy in all eligible patients $(n=40)$

Grade 1 Grade 2 Grade 3 Grade $4 \%$ grade 3/4

\begin{tabular}{lrrrll}
\hline \multicolumn{2}{l}{ Laboratory findings } & & & \\
Leukocyte & 9 & 19 & 8 & 3 & 27.5 \\
Neutrophil & 7 & 10 & 16 & 6 & 55.0 \\
Hemoglobin & 12 & 18 & 5 & 0 & 12.5 \\
Platelet & 25 & 2 & 0 & 0 & 0 \\
Total bilirubin & 6 & 1 & 0 & 0 & 0 \\
AST & 6 & 1 & 0 & 0 & 0 \\
ALT & 8 & 1 & 0 & 0 & 0 \\
Creatinine & 16 & 4 & 0 & 0 & 0 \\
Hyponatremia & 14 & 0 & 8 & 1 & 22.5 \\
Febrile neutro- & 0 & 0 & 3 & 0 & 7.5 \\
$\quad$ penia & & & & & \\
Objective findings & & & & & \\
Fatigue & 9 & 2 & 0 & 0 & 0 \\
Anorexia & 12 & 13 & 8 & 0 & 20.0 \\
Diarrhea & 5 & 2 & 4 & 0 & 10.0 \\
Nausea & 12 & 8 & 6 & 0 & 15.0 \\
Vomiting & 8 & 5 & 0 & 0 & 0 \\
Stomatitis & 4 & 4 & 0 & 0 & 0 \\
\hline
\end{tabular}

Table 3 Surgical findings in 40 patients

\begin{tabular}{lc}
\hline & Values \\
\hline Gastrectomy & $10(25 \%)$ \\
Distal & $30(75 \%)$ \\
Total & \\
Lymph node dissection & $1(3 \%)$ \\
D1 & $1(3 \%)$ \\
D1+ & $38(95 \%)$ \\
D2 & $325(230-525)$ \\
Operation time (min) & \\
Blood loss (mL) & \\
Blood transfusion & $725(160-2200)$ \\
Yes & $16(40 \%)$ \\
No & $24(60 \%)$ \\
\hline
\end{tabular}

${ }^{a}$ The median value is given, with the range in parentheses

for advanced or recurrent gastric cancer and concluded that DCS was a well-tolerated and highly active regimen, with an overall RR of $81 \%$ [6]. Based on these findings, we conducted the present trial.

The reasons why we adopted $\mathrm{R} 0$ resection rate as the primary endpoint is as follows: (1) the primary endpoint of JCOG0405 was R0 resection rate. (2) Similar NAC trials such as JCOG0001, JCOG0210, and JCOG0405 have data of $\mathrm{R} 0$ resection rate which can be used as criteria to determine the efficacy of the present study. (3) R0 resection
Table 4 Pathological findings for 40 patients

\begin{tabular}{|c|c|}
\hline & Values \\
\hline \multicolumn{2}{|l|}{ Tumor depth } \\
\hline урТ0 & $3(8 \%)$ \\
\hline урТ1 & $2(5 \%)$ \\
\hline урТ2 & $4(10 \%)$ \\
\hline урт3 & $9(23 \%)$ \\
\hline урт4 & $22(55 \%)$ \\
\hline \multicolumn{2}{|l|}{ Lymph node metastases } \\
\hline ypNO & $15(38 \%)$ \\
\hline ypN1 & $9(23 \%)$ \\
\hline ypN2 & $5(13 \%)$ \\
\hline ypN3a, ypN3b & $11(28 \%)$ \\
\hline Median number of retrieved lymph nodes ${ }^{a}$ & $37(13-82)$ \\
\hline \multicolumn{2}{|l|}{ Peritoneal disease } \\
\hline Yes & $2(5 \%)$ \\
\hline \multicolumn{2}{|l|}{ M category } \\
\hline M0 & $38(95 \%)$ \\
\hline ypM1 & $2(5 \%)$ \\
\hline \multicolumn{2}{|l|}{ Resection } \\
\hline R0 & $36(90 \%)$ \\
\hline $\mathrm{R} 1$ & $4(10 \%)$ \\
\hline \multicolumn{2}{|l|}{ ypStage } \\
\hline IB & $3(8 \%)$ \\
\hline IIA & $3(8 \%)$ \\
\hline IIB & $13(33 \%)$ \\
\hline IIIA & $3(8 \%)$ \\
\hline IIIB & $4(10 \%)$ \\
\hline IIIC & $9(23 \%)$ \\
\hline IV & $2(5 \%)$ \\
\hline Unclassifiable & $3(8 \%)$ \\
\hline \multicolumn{2}{|l|}{ Histological type } \\
\hline Differentiated & $8(20 \%)$ \\
\hline Undifferentiated & $27(68 \%)$ \\
\hline Other & $5(13 \%)$ \\
\hline \multicolumn{2}{|l|}{ Lymphatic invasion } \\
\hline Yes & $21(52 \%)$ \\
\hline \multicolumn{2}{|l|}{ Vascular invasion } \\
\hline Yes & $24(60 \%)$ \\
\hline \multicolumn{2}{|l|}{ Pathological response } \\
\hline Grade 0 & $4(10 \%)$ \\
\hline Grade 1a & $13(33 \%)$ \\
\hline Grade $1 b$ & $13(33 \%)$ \\
\hline Grade 2 & $7(18 \%)$ \\
\hline Grade 3 & $3(8 \%)$ \\
\hline
\end{tabular}

${ }^{\mathrm{a}}$ The range is given in parentheses

rate becomes evident immediately after surgery, whereas the true endpoint of 3-year overall survival rate does not become evident for 3 years after the registration of the last patient. If 3-year overall survival rate was selected as the 
Table 5 Morbidity after surgery in 40 patients

\begin{tabular}{llllll}
\hline & \multicolumn{3}{l}{ Grade $^{\mathrm{a}}$} & & Grade 3-4 (\%) \\
\cline { 2 - 4 } & 1 & 2 & 3 & 4 & \\
\hline Pancreatic fistula & 0 & 0 & 5 & 0 & 12.5 \\
Abdominal infection & 0 & 2 & 0 & 0 & 0 \\
Anastomotic leak & 0 & 0 & 1 & 0 & 2.5 \\
Anastomotic stricture & 0 & 0 & 1 & 0 & 2.5 \\
Small-intestinal obstruction & 0 & 0 & 2 & 0 & 5 \\
Ileus & 0 & 1 & 0 & 0 & 0 \\
Pleural effusion & 5 & 1 & 0 & 0 & 0 \\
Chylous ascites & 1 & 0 & 0 & 0 & 0
\end{tabular}

${ }^{a}$ National Cancer Institute Common Terminology Criteria for Adverse Events version 3.0

primary endpoint and $\mathrm{R} 0$ resection rate was not better than the threshold value of $65 \%$, more toxic and not effective regimen of DCS therapy could not be denied for 3 years.

$\mathrm{R} 0$ resection rate in the present study was $90 \%$ which is much higher than that of the JCOG0210 (73\%) where they did not perform staging laparoscopy or JCOG0501 (74.2\%), where they included CY1 and even P1 near the primary gastric cancer. $\mathrm{R} 0$ resection rates of the patients with bulky $\mathrm{N} 2$ lymph nodes and those with large type 3 or type 4 tumors were $100 \%$ (7/7) and $88 \%$ (30/34) in the present study, respectively. As we initially excluded patients with CY1 at the staging laparoscopy until the protocol amendment in 2013 , only 4 patients finally had positive peritoneal cytology test at the initial staging laparoscopy and such exclusion of patients with CY1 should have played a critical role in achieving this high $\mathrm{R} 0$-resection rate. Moreover, inclusion of bulky $\mathrm{N} 2$ and administration of as many as 4 cycles of the DCS therapy instead of 2 cycles may also have contributed to this high $\mathrm{R} 0$ resection rate.

JCOG1002 was planned as a phase II trial with the expectation that neoadjuvant DCS would be more effective than neoadjuvant CS in terms of tumor shrinkage. This trial evaluated the efficacy of preoperative chemotherapy for not only bulky N2 lymph node metastasis but also para-aortic lymph node metastasis [13]. Contrary to expectation, the primary endpoint, the clinical RR, was $57.7 \%$, which was lower than the predefined threshold rate of $65 \%$. Therefore, preoperative DCS chemotherapy cannot be a standard treatment for gastric cancer with ELM. The cohort in the present trial comprised patients with large type 3 or type 4 tumors, in addition to cancer with bulky N2 lymph nodes. Seven patients had bulky N2 lymph nodes; their clinical RR was $71.4 \%$ (5/7). All five patients with bulky N2 lymph nodes showing a clinical response received 4 cycles of DCS NAC. Unlike CS therapy, more than 2 cycles of DCS therapy might be needed to achieve a good response. The 2 patients without a clinical response, as defined by the Response Evaluation
Criteria in Solid Tumors (RECIST) 1.0, had received 4 and 2 cycles of DCS NAC.

The JCOG considers gastric cancer with para-aortic lymph node metastasis as resectable and patients as candidates for NAC. In the JCOG0405 study, the 5-year survival rate of patients with ELM after CF NAC followed by surgery was $53 \%$. Therefore, this treatment strategy is a possible standard modality for gastric cancer with ELM. Meanwhile, para-aortic lymph nodes were not classified as regional lymph nodes in the Japanese classification of gastric carcinoma [8]. We considered that para-aortic metastasis is a distant metastasis and developed an original treatment strategy for this type of cancer [14]. This is why we excluded patients with para-aortic lymph node metastasis from the present study.

The JCOG considers type 4 and large type 3 tumors as a distinct category from gastric cancer with ELM and has developed treatment strategies that are different from those for gastric cancer with ELM. In JCOG0210, patients received CS chemotherapy followed by surgery; this trial showed a good 3-year survival rate of $24.5 \%$ [3]; thus, the next phase III study (JCOG0501) was conducted. However, S-1 adjuvant chemotherapy for 1 year showed remarkable survival benefits for type 4 or large type 3 gastric cancer, while additional NAC with cisplatin and S-1 did not demonstrate any additional benefit to $S-1$ adjuvant chemotherapy [15]. Docetaxel, cisplatin, and 5-FU (key drug of S-1) have different antitumor activities, which are involved in tubulin depolymerization, DNA cross-linking, and DNA metabolism, respectively, and the synergistic effects were recognized by concurrent administration to mammary tumor cells in an experimental animal model [16]. Reflecting this experimental result, docetaxel/cisplatin/5-FU actually has been demonstrated to improve patient survival compared to cisplatin/5-FU in squamous-cell carcinoma such as head and neck cancer [17] and esophageal cancer [18] in the NAC setting. Nevertheless, there have not been such reports with regard to adenocarcinoma. Although the significance as NAC in the SP regimen was denied in the JCOG 0501 trial for large type 3 and type 4 gastric cancers, the DCS regimen might have a given potential to improve patient survival in the specific gastric cancer through the biological rationale. In fact, the $\mathrm{R} 0$ resection rate of the present study is very high. However, pathological RR is only $57.5 \%$ which is not much different from past clinical trials of JCOG0405 (51\%), JCOG0210 (47\%), and JCOG0501 (51\%). Whether DCS NAC could extend survival longer than CS NAC is unknown. This relatively low rate of pathological response might be predicting long-term outcomes not to reach the expected level. The long-term outcomes of the patient panel included in the present study should be assessed.

Recently, a phase II study of NAC with docetaxel, oxaliplatin, and S-1 showed that this regimen was safe 
and effective for resectable advanced gastric cancer, with a R0 resection rate of $97.6 \%$ [19]. In addition, perioperative chemotherapy with fluorouracil/leucovorin, oxaliplatin, and docetaxel improved overall survival when compared to perioperative chemotherapy with ECF or epirubicin, cisplatin, and capecitabine in a randomized phase III trial [20]. Similar to cisplatin, oxaliplatin is also classified as a platinum-based anticancer agent. However, oxaliplatin is thought to have lower renal toxicity than cisplatin. Therefore, oxaliplatin rather than cisplatin may play a key role in the development of NAC for resectable advanced gastric cancer.

As a postoperative adjuvant chemotherapy, docetaxel/S-1 was superior to S-1 alone for pathological Stage III gastric cancer in the JACCRO GC-07 trial [21]. JACCRO GC-07 trial included patients undergoing R0 resection. Meanwhile, the present study included patients with tumors that were unclear whether R0 resection was possible or not. The subjects were different between the studies. It was reported that large type 3 and type 4 gastric cancers accounted for only about $10 \%$ of stage II/III gastric cancer and large type 3 or type 4 gastric cancer was a poor prognostic factor independent of pathological stage [11]. Even if docetaxel/S-1 is superior to $\mathrm{S}-1$ alone for pathological stage III gastric cancer, the same would not necessarily be true for the present research subject.

There are some limitations to the present study. First, the present trial had a single-arm, phase II design and was conducted at a single institution. Second, only short-term outcomes were assessed. Third, the accrual period was quite long; therefore chemotherapy regimens after progression varied during the study period. Initially, patient accrual was determined to be from February 2010 to February 2014. However, patient accrual was so poor that we amended the study protocol to broaden the eligibility criteria.

In conclusion, DCS NAC was feasible and showed a sufficient $\mathrm{R} 0$ resection rate in resectable advanced gastric cancer with type 4 / large type 3 tumors or bulky N2 lymph nodes. Long-term survival outcomes should be assessed for the present study population.

Acknowledgements We would like to thank Editage (http://www.edita ge.jp) for English language editing.

\section{Compliance with ethical standards}

Conflict of interest The authors declare that they have no conflict of interest.

Human rights statement and informed consent All procedures followed were in accordance with the ethical standards of the responsible committee on human experimentation (institutional and national) and with the Helsinki Declaration of 1964 and later versions. Informed consent or substitute for it was obtained from all patients for being included in the study.

\section{References}

1. Ferlay J, Soerjomataram I, Dikshit R, Eser S, Mathers C, Rebelo $\mathrm{M}$, et al. Cancer incidence and mortality worldwide: sources, methods and major patterns in GLOBOCAN 2012. Int J Cancer. 2015;136:E359-86.

2. Yoshikawa T, Sasako M, Yamamoto S, Sano T, Imamura H, Fujitani K, et al. Phase II study of neoadjuvant chemotherapy and extended surgery for locally advanced gastric cancer. Br J Surg. 2009;96:1015-22.

3. Iwasaki Y, Sasako M, Yamamoto S, Nakamura K, Sano T, Katai $\mathrm{H}$, et al. Phase II study of preoperative chemotherapy with S-1 and cisplatin followed by gastrectomy for clinically resectable type 4 and large type 3 gastric cancers (JCOG0210). J Surg Oncol. 2013;107:741-5.

4. Tsuburaya A, Mizusawa J, Tanaka Y, Fukushima N, Nashimoto A, Sasako M. Neoadjuvant chemotherapy with S-1 and cisplatin followed by $\mathrm{D} 2$ gastrectomy with para-aortic lymph node dissection for gastric cancer with extensive lymph node metastasis. $\mathrm{Br}$ J Surg. 2014;101:653-60.

5. Van Cutsem E, Moiseyenko VM, Tjulandin S, Majlis A, Constenla $\mathrm{M}$, Boni C, et al. Phase III study of docetaxel and cisplatin plus fluorouracil compared with cisplatin and fluorouracil as first-line therapy for advanced gastric cancer: a report of the V325 Study Group. J Clin Oncol. 2006;24:4991-7.

6. Koizumi W, Nakayama N, Tanabe S, Sasaki T, Higuchi K, Nishimura K, et al. A multicenter phase II study of combined chemotherapy with docetaxel, cisplatin, and S-1 in patients with unresectable or recurrent gastric cancer (KDOG 0601). Cancer Chemother Pharmacol. 2012;69:407-13.

7. Japanese gastric cancer. Treatment guidelines 2010 (ver. 3). Gastric Cancer. 2011;14:113-23.

8. Japanese classification of gastric carcinoma. 3rd English edition. Gastric Cancer. 2011;14:101-12.

9. Li C, Oh SJ, Kim S, Hyung WJ, Yan M, Zhu ZG, et al. Macroscopic Borrmann type as a simple prognostic indicator in patients with advanced gastric cancer. Oncology. 2009;77:197-204.

10. Yamashita K, Sakuramoto S, Katada N, Kikuchi S, Watanabe M. Simple prognostic indicators using macroscopic features and age in advanced gastric cancer. Hepatogastroenterology. 2014;61:512-7.

11. Yamashita K, Ema A, Hosoda K, Mieno H, Moriya H, Katada N, et al. Macroscopic appearance of Type IV and giant Type III is a high risk for a poor prognosis in pathological stage II/III advanced gastric cancer with postoperative adjuvant chemotherapy. World J Gastrointest Oncol. 2017;9:166-75.

12. Cunningham D, Allum WH, Stenning SP, Thompson JN, Van de Velde CJ, Nicolson M, et al. Perioperative chemotherapy versus surgery alone for resectable gastroesophageal cancer. N Engl J Med. 2006;355:11-20.

13. Ito S, Sano T, Mizusawa J, Takahari D, Katayama H, Katai $\mathrm{H}$, et al. A phase II study of preoperative chemotherapy with docetaxel, cisplatin, and S-1 followed by gastrectomy with D2 plus para-aortic lymph node dissection for gastric cancer with extensive lymph node metastasis: JCOG1002. Gastric Cancer. 2017;20:322-31.

14. Mieno H, Yamashita K, Hosoda K, Moriya H, Higuchi K, Azuma $\mathrm{M}$, et al. Conversion surgery after combination chemotherapy of docetaxel, cisplatin and S-1 (DCS) for far-advanced gastric cancer. Surg Today. 2017;47:1249-58.

15. Iwasaki Y, Terashima M, Mizusawa J, Katayama H, Nakamura $\mathrm{K}$, Katai H, et al. Randomized phase III trial of gastrectomy with or without neoadjuvant S-1 plus cisplatin for type 4 or large type 3 gastric cancer: Japan Clinical Oncology Group study (JCOG0501). Am Soc Clin Oncol. 2018;2018:4046 
16. Bissery MC, Vrignaud P, Lavelle F. Preclinical profile of docetaxel (taxotere): efficacy as a single agent and in combination. Semin Oncol. 1995;22:3-16.

17. Posner MR, Hershock DM, Blajman CR, Mickiewicz E, Winquist E, Gorbounova V, et al. Cisplatin and fluorouracil alone or with docetaxel in head and neck cancer. N Engl J Med. 2007;357:1705-15.

18. Yamashita K, Hosoda K, Moriya H, Katada C, Sugawara M, Mieno H, et al. Prognostic advantage of docetaxel/cisplatin/ 5 -fluorouracil neoadjuvant chemotherapy in clinical stage II/III esophageal squamous cell carcinoma due to excellent control of preoperative disease and postoperative lymph node recurrence. Oncology. 2017;92:221-8.

19. Park I, Ryu MH, Choi YH, Kang HJ, Yook JH, Park YS, et al. A phase II study of neoadjuvant docetaxel, oxaliplatin, and S-1 (DOS) chemotherapy followed by surgery and adjuvant S-1 chemotherapy in potentially resectable gastric or gastroesophageal junction adenocarcinoma. Cancer Chemother Pharmacol. 2013;72:815-23.

20. Al-Batran S-E, Homann N, Schmalenberg H, Kopp H-G, Haag GM, Luley KB, et al. Perioperative chemotherapy with docetaxel, oxaliplatin, and fluorouracil/leucovorin (FLOT) versus epirubicin, cisplatin, and fluorouracil or capecitabine (ECF/ECX) for resectable gastric or gastroesophageal junction (GEJ) adenocarcinoma (FLOT4-AIO): a multicenter, randomized phase 3 trial. J Clin Oncol. 2017;35:4004.

21. Kodera Y, Yoshida K, Kochi M, Ichikawa W, Kakeji Y, Sano T, et al. A randomized phase III study comparing S-1 plus docetaxel with $\mathrm{S}-1$ alone as a postoperative adjuvant chemotherapy for curatively resected stage III gastric cancer (JACCRO GC-07 trial). J Clin Oncol. 2018;36:4007. 